\title{
The eventual paracanonical map of a variety of maximal Albanese dimension
}

\author{
Miguel Ángel Barja, Rita Pardini and Lidia Stoppino
}

\begin{abstract}
Let $X$ be a smooth complex projective variety such that the Albanese map of $X$ is generically finite onto its image. Here, we study the so-called eventual $m$-paracanonical map of $X$, whose existence is implied by previous results of the authors (when $m=1$, we also assume $\chi\left(K_{X}\right)>0$ ). We show that for $m=1$, this map behaves in a similar way to the canonical map of a surface of general type, as described by Beauville, while it is birational for $m>1$. We also describe it explicitly in several examples.
\end{abstract}

\section{Introduction}

Let $X$ be a smooth complex projective variety, and let $a: X \rightarrow A$ be a map to an abelian variety that is generically finite onto its image and such that $a^{*}: \operatorname{Pic}^{0}(A) \rightarrow \operatorname{Pic}^{0}(X)$ is injective; we identify $\operatorname{Pic}^{0}(A)$ with a subgroup of $\operatorname{Pic}^{0}(X)$ via $a^{*}$.

Let $L \in \operatorname{Pic}(X)$ be a line bundle such that $h^{0}(L \otimes \alpha)>0$ for every $\alpha \in \operatorname{Pic}^{0}(A)$. We may consider the pull-back $L^{(d)}$ to the étale cover $X^{(d)} \rightarrow X$ induced by the $d$ th multiplication map of $A$. In [BPS16a], it is shown that for $d$ large and divisible enough and $\alpha \in \operatorname{Pic}^{0}(A)$ general, the map given by $\left|L^{(d)} \otimes \alpha\right|$ does not depend on $\alpha$ and is obtained by base change from the so-called eventual map associated with $L$, a generically finite map $\phi: X \rightarrow Z$, uniquely determined up to birational isomorphism, such that $a$ factors through it.

Here we consider the case when $a: X \rightarrow A$ is the Albanese map and $L=m K_{X}$, with $m \geqslant 1$, and study the eventual $m$-paracanonical map, which is intrinsically attached to $A$. (For $m=1$ one needs to also assume $\chi\left(K_{X}\right)>0$, since by the generic vanishing theorem, $\chi\left(K_{X}\right)$ is the generic value of $h^{0}\left(K_{X} \otimes \alpha\right)$.)

After recalling (Section 2) the main properties of the eventual map, in Section 3, we concentrate on the case $m=1$ and prove that the relations between the numerical invariants of $X$ and of the paracanonical image $Z$ are completely analogous to those between the numerical invariants of a surface of general type and its canonical image (see [Bea79]). In Section 4, we prove that

Received 5 March 2017, accepted in final form 19 January 2018.

2010 Mathematics Subject Classification 14C20, 14J29, 14J30, 14J35, 14J40.

Keywords: irregular variety, variety of maximal Albanese dimension, eventual map, paracanonical map, pluricanonical map.

This journal is (C) Foundation Compositio Mathematica 2019. This article is distributed with Open Access under the terms of the Creative Commons Attribution Non-Commercial License, which permits non-commercial reuse, distribution, and reproduction in any medium, provided that the original work is properly cited. For commercial re-use, please contact the Foundation Compositio Mathematica.

The first author was supported by MINECO MTM2015-69135-P "Geometría y Topología de Variedades, Álgebra y Aplicaciones" and by Generalitat de Catalunya SGR2014-634. The second and third author are members of G.N.S.A.G.A.-I.N.d.A.M. This research was partially supported by MIUR (Italy) through PRIN 2015 "Geometria delle varietà algebriche" and PRIN 2012-13 "Moduli, strutture geometriche e loro applicazioni". 
the eventual $m$-canonical map is always birational for $m>1$, and therefore $m=1$ is the only interesting case.

Section 5 contains several examples that show that the eventual paracanonical map is really a new object, need not be birational or coincide with the Albanese map and can have arbitrarily large degree. We finish by posing a couple of questions.

Conventions. We work over the complex numbers.

In this paper, the focus is on birational geometry, so a map is a rational map, and we denote all maps by solid arrows.

Given maps $f: X \rightarrow Y$ and $g: X \rightarrow Z$, we say that $g$ is composed with $f$ if there exists a map $h: Y \rightarrow Z$ such that $g=h \circ f$. Given a map $f: X \rightarrow Y$ and an involution $\sigma$ of $X$, we say that $f$ is composed with $\sigma$ if $f \circ \sigma=f$.

We say that two dominant maps $f: X \rightarrow Z$ and $f^{\prime}: X \rightarrow Z^{\prime}$ are birationally equivalent if there exists a birational isomorphism $h: Z \rightarrow Z^{\prime}$ such that $f^{\prime}=h \circ f$.

A generically finite map means a rational map that is generically finite onto its image. A smooth projective variety $X$ is of maximal Albanese dimension if its Albanese map is generically finite.

The symbol $\equiv$ denotes numerical equivalence of line bundles or $\mathbb{Q}$-divisors.

\section{Preliminaries}

In this section, we fix the setup and recall some facts and definitions. More details can be found in [Bar15, BPS16b, BPS16a].

Let $X$ be a smooth projective map, and let $a: X \rightarrow A$ be a generically finite map to an abelian variety of dimension $q$. Given a line bundle $L$ on $X$, the continuous rank $h_{a}^{0}(L)$ of $L$ (with respect to $a$ ) is defined as the minimum value of $h^{0}(L \otimes \alpha)$ as $\alpha$ varies in $\operatorname{Pic}^{0}(A)$.

Let $d$ be an integer, and denote by $\mu_{d}: A \rightarrow A$ the multiplication by $d$; the following cartesian diagram defines the variety $X^{(d)}$ and the maps $a_{d}$ and $\widetilde{\mu_{d}}$ :

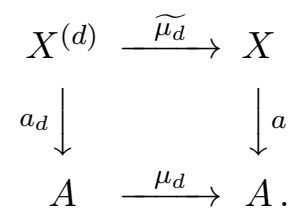

We set $L^{(d)}:={\widetilde{\mu_{d}}}^{*} L$; one has $h_{a}^{0}\left(L^{(d)}\right)=d^{2 q} h_{a}^{0}(L)$.

We say that $a$ is strongly generating if the induced map $\operatorname{Pic}^{0}(A) \rightarrow \operatorname{Pic}^{0}(X)$ is an inclusion; notice that if $a$ is strongly generating, then the variety $X^{(d)}$ is connected for every $d$.

DEFINITION 2.1. We say that a certain property holds generically for $L$ (with respect to $a$ ) if and only if it holds for $L \otimes \alpha$ for general $\alpha \in \operatorname{Pic}^{0}(A)$; similarly, we say that a property holds eventually (with respect to $a$ ) for $L$ if and only if it holds for $L^{(d)}$ for $d$ sufficiently large and divisible. For instance, we say that $L$ is eventually generically birational if for $d$ sufficiently large and divisible, $L^{(d)} \otimes \alpha$ is birational for $\alpha \in \operatorname{Pic}^{0}(A)$ general.

In [BPS16a], we have studied the eventual generic behaviour of a line bundle $L$ with $h_{a}^{0}(L)>0$. THEOREM 2.2 [BPS16a, Theorem 3.7]. Let $X$ be a smooth projective variety with a strongly generating and generically finite map $a: X \rightarrow A$ to an abelian variety $A$, and let $L \in \operatorname{Pic}(X)$ be such that $h_{a}^{0}(L)>0$. 


\section{M. Á. Barja, R. PArdini And L. Stoppino}

Then there exists a generically finite map $\varphi: X \rightarrow Z$, unique up to birational equivalence, such that

(i) the map $a: X \rightarrow A$ is composed with $\varphi$;

(ii) if, for $d \geqslant 1$, we denote by $\varphi^{(d)}: X^{(d)} \rightarrow Z^{(d)}$ the map obtained from $\varphi: X \rightarrow Z$ by taking base change with $\mu_{d}$, then the map given by $\left|L^{(d)} \otimes \alpha\right|$ is composed with $\varphi^{(d)}$ for $\alpha \in \operatorname{Pic}^{0}(A)$ general;

(iii) for $d$ sufficiently large and divisible, the map $\varphi^{(d)}$ is birationally equivalent to the map given by $\left|L^{(d)} \otimes \alpha\right|$ for $\alpha \in \operatorname{Pic}^{0}(A)$ general.

The map $\varphi: X \rightarrow Z$ is called the eventual map given by $L$, and the degree $m_{L}$ of $\varphi$ is called the eventual degree of $L$.

\section{Eventual behaviour of the paracanonical system}

With the same notation as in Section 2, we assume that $X$ is a smooth projective variety of maximal Albanese dimension with $\chi\left(K_{X}\right)>0$, and we consider $L=K_{X}$. Since $h_{a}^{0}\left(K_{X}\right)=\chi\left(K_{X}\right)$ by generic vanishing, Theorem 2.2 can be applied to $L=K_{X}$, taking as $a: X \rightarrow A$ the Albanese map of $X$.

We call the eventual map $\varphi: X \rightarrow Z$ given by $K_{X}$ the eventual paracanonical map, its image $Z$ the eventual paracanonical image and its degree $m_{X}:=m_{K_{X}}$ the eventual paracanonical degree.

The following result is analogous to [Bea79, Theorem 3.1].

THEOREM 3.1. In the above setup, denote by $\widetilde{Z}$ any smooth model of $Z$. Then one of the following occurs:

(i) $\chi\left(K_{\widetilde{Z}}\right)=\chi\left(K_{X}\right)$.

(ii) $\chi\left(K_{\widetilde{Z}}\right)=0$.

Furthermore, in case (b), the Albanese image of $X$ is ruled by tori.

Proof. We adapt the proof of [Bea79, Theorem 3.1].

Assume $\chi\left(K_{\widetilde{Z}}\right)>0$. Up to replacing $X$ by a suitable smooth birational model, we may assume that the induced map $\varphi: X \rightarrow \widetilde{Z}$ is a morphism. Recall that by Theorem 2.2, the Albanese map of $X$ is composed with $\varphi$. Fix $\alpha \in \operatorname{Pic}^{0}(A)$ general, so that, in particular, the map given by $\left|K_{X} \otimes \alpha\right|$ is composed with $\varphi$.

Pick $0 \neq \omega \in H^{0}\left(K_{\widetilde{Z}} \otimes \alpha\right)$, denote by $\varphi^{*} \omega \in H^{0}\left(K_{X} \otimes \alpha\right)$ the pull-back of $\omega$ regarded as a differential form with values in $\alpha$, and set $D:=\operatorname{div}(\omega)$. The Hurwitz formula gives $\operatorname{div}\left(\varphi^{*} \omega\right)=$ $\varphi^{*} D+R$, where $R$ is the ramification divisor of $\varphi$. On the other hand, by assumption, we have $\operatorname{div}\left(\varphi^{*} \omega\right)=\varphi^{*} H_{0}+F$, where $H_{0}$ is an effective divisor of $\widetilde{Z}$ and $F$ is the fixed part of $\left|K_{X} \otimes \alpha\right|$. Hence, we have

$$
\varphi^{*} D+R=\varphi^{*} H_{0}+F .
$$

We want to use the above relation to show that $H_{0} \leqslant D$. Let $\Gamma$ be a prime divisor of $\widetilde{Z}$, let $\Gamma^{\prime}$ be a component of $\varphi^{*} \Gamma$, and let $e$ be the ramification index of $\varphi$ along $\Gamma^{\prime}$; that is, $e$ is the multiplicity of $\Gamma^{\prime}$ in $\varphi^{*} \Gamma$. Note that $\Gamma^{\prime}$ appears in $R$ with multiplicity $e-1$. If $a$ is the multiplicity of $\Gamma$ in $D$ and $b$ is the multiplicity of $\Gamma$ in $H_{0}$, then comparing the multiplicity of $\Gamma^{\prime}$ in both sides of (3.1), we get $e a+e-1 \geqslant e b$, namely $a \geqslant b$. This shows that $H_{0} \leqslant D$, and we have the following chain 
of inequalities:

$$
h^{0}\left(H_{0}\right) \leqslant h^{0}\left(K_{\widetilde{Z}} \otimes \alpha\right) \leqslant h^{0}\left(K_{X} \otimes \alpha\right)=h^{0}\left(H_{0}\right) .
$$

So all the inequalities in (3.2) are actually equalities and, by the generality of $\alpha$, we have $\chi\left(K_{\widetilde{Z}}\right)=$ $h^{0}\left(K_{\widetilde{Z}} \otimes \alpha\right)=h^{0}\left(K_{X} \otimes \alpha\right)=\chi\left(K_{X}\right)$.

Assume $\chi\left(K_{\widetilde{Z}}\right)=0$, instead. Then the Albanese image of $\widetilde{Z}$ is ruled by tori by [EL97, Theorem 3]. Let $\beta: \widetilde{Z} \rightarrow A$ be the map such that $a=\beta \circ \varphi$. By the universal property of the Albanese map, $\beta$ is the Albanese map of $\widetilde{Z}$; hence, $a(X)=\beta(\widetilde{Z})$ is also ruled by tori.

Corollary 3.2. In the above setup, denote by $n$ the dimension of $X$. Then

(i) $\operatorname{vol}\left(K_{X}\right) \geqslant m_{X} n ! \chi\left(K_{X}\right)$;

(ii) if $\chi\left(K_{\widetilde{Z}}\right)>0$, then $\operatorname{vol}\left(K_{X}\right) \geqslant 2 m_{X} n ! \chi\left(K_{X}\right)$.

In particular, the stronger inequality (ii) holds whenever the Albanese image of $X$ is not ruled by tori.

Proof. Part (i) follows directly by [BPS16a, Theorem 6.8].

For part (ii), observe that if $\chi\left(K_{\widetilde{Z}}\right)>0$, then $\chi\left(K_{X}\right)=\chi\left(K_{\widetilde{Z}}\right)$ by Theorem 3.1 ; the main theorem of [Bar15] gives $\operatorname{vol}\left(K_{\widetilde{Z}}\right) \geqslant 2 n ! \chi\left(K_{\widetilde{Z}}\right)=2 n ! \chi\left(K_{X}\right)$ and, arguing as in Corollary 3.12, we have inequality (ii).

Finally, the last sentence in the statement is a consequence of Theorem 3.1.

One can be more precise in the surface case.

Proposition 3.3. In the above setup, assume $\operatorname{dim} X=2$ and that $X$ is minimal of general type. Then one of the following cases occurs:

(i) We have $p_{g}(X)=p_{g}(\widetilde{Z}), q(X)=q(\widetilde{Z})$ and $m_{X} \leqslant 2$.

(ii) We have $\chi(\widetilde{Z})=0$ and $2 \leqslant m_{X} \leqslant 4$, and the Albanese image of $X$ is ruled by tori.

Proof. Assume $\chi(\widetilde{Z})>0$; then by Theorem 3.1, we have $\chi(\widetilde{Z})=\chi(X)$, and Corollary 3.2(ii) gives $K_{X}^{2} \geqslant 4 m_{X} \chi(X)$. Hence, $m_{X} \leqslant 2$ follows immediately by the Bogomolov-Miyaoka-Yau inequality $K_{X}^{2} \leqslant 9 \chi\left(K_{X}\right)$. We have $q(\widetilde{Z})=q(X)$ since the Albanese map of $X$ is composed with $\varphi: X \rightarrow \widetilde{Z}$, so $p_{g}(X)=\chi(X)+q(X)-1=\chi(\widetilde{Z})+q(\widetilde{Z})-1=p_{g}(\widetilde{Z})$.

Now, assume $\chi(\widetilde{Z})=0$; in this case, the Albanese image of $X$ is ruled by tori by Theorem 3.1, and Corollary 3.2(i) gives $K_{X}^{2} \geqslant 2 m_{X} \chi(X)$. Using the Bogomolov-Miyaoka-Yau inequality as above, we get $m_{X} \leqslant 4$. On the other hand, $m_{X}>1$ since $\varphi$ cannot be birational because $\chi(X)>\chi(\widetilde{Z})$.

Remark 3.4. For the case of a minimal smooth threefold of general type $X$, it is proven in [CCZ07] that the inequality $K_{X}^{3} \leqslant 72 \chi(X)$ holds. Hence, the arguments used above to prove Proposition 3.3 show that in this case, we have $m_{X} \leqslant 6$ if $\chi(\widetilde{Z})>0$ and $m_{X} \leqslant 12$ if $\chi(\widetilde{Z})=0$.

\section{Eventual behaviour of the $m$-paracanonical system for $m>1$}

In this section, we consider the eventual $m$-paracanonical map for varieties of general type and maximal Albanese dimension, for $m \geqslant 2$. Our main result implies that for $m \geqslant 2$, the eventual $m$-paracanonical map does not give additional information on the geometry of $X$. 


\section{M. Á. Barja, R. PArdini And L. Stoppino}

THEOREM 4.1. Let $X$ be a smooth projective $n$-dimensional variety of general type and maximal Albanese dimension; denote the Albanese map by $a: X \rightarrow A$.

Then there exists a positive integer $d$ such that the system $\left|m K_{X^{(d)}} \otimes \alpha\right|$ is birational for every $m \geqslant 2$ and every $\alpha \in \operatorname{Pic}^{0}(A)$. In particular, $m K_{X}$ is eventually generically birational for $m \geqslant 2$.

Remark 4.2. The question whether the $m$-canonical system of a variety of maximal Albanese dimension is birational has been considered by several authors.

The answer is positive for $m \geqslant 3$ [JLT13, CH02, PP11].

The case $m=2$ has been studied in [BLNP12] for a variety $X$ of maximal Albanese dimension with $q(X)>\operatorname{dim} X$. The authors prove that if $\left|2 K_{X}\right|$ is not birational, then either $X$ is birational to a theta divisor in a principally polarized abelian variety (p.p.a.v.), or there exists a fibration $f: X \rightarrow Y$ onto an irregular variety with $\operatorname{dim} Y<\operatorname{dim} X$. If $X$ is a smooth theta divisor in a p.p.a.v., then it is easy to check directly that $\left|2 K_{X} \otimes \alpha\right|$ is birational for $0 \neq \alpha \in \operatorname{Pic}^{0}(X)$ and that for every $d \geqslant 2$, the system $\left|K_{X^{(d)}} \otimes \alpha\right|$ is birational for every $\alpha \in \operatorname{Pic}^{0}(X)$.

Warning. In this section, in accordance with the standard use in birational geometry, we switch to the additive notation and write $L+\alpha$ instead of $L \otimes \alpha$.

We fix a very ample divisor $H$ on $A$, and we set $M:=a^{*} H$; we use the notation of dia$\operatorname{gram}(2.1)$ and set $M_{d}:=a_{d}^{*} H$ for $d>1$. By [BL04, Proposition 2.3.5], we have $\widetilde{\mu_{d}}{ }^{*} M \equiv d^{2} M_{d}$.

Moreover, since the question is birational, we may assume that the map $f: X \rightarrow Y$ to the canonical model (which exists by [BCHM10]) is a log resolution.

To prove Theorem 4.1, we use the following preliminary result, that generalizes [BPS16b, Lemma 2.4].

Lemma 4.3. In the above setup, there exists a $d \gg 0$ such that

$$
K_{X^{(d)}}=L+n M_{d}+E,
$$

where $L$ and $E$ are $\mathbb{Q}$-divisors such that $L$ is nef and big and $E$ is effective with normal crossings support.

Proof. By [HM07, Corollary 1.5], the fibres of $f$ are rationally chain connected; hence, they are contracted to points by $a$; it follows that the Albanese map of $X$ descends to a morphism $\bar{a}: Y \rightarrow A$.

Let $\bar{M}:=\bar{a}^{*} H$. There exists an $\epsilon>0$ such that for $|t|<\epsilon$, the class $K_{Y}-t \bar{M}$ is ample and therefore $f^{*} K_{Y}-t M$ is nef and big on $X$. By the definition of the canonical model, we have $K_{X}=f^{*} K_{Y}+E$, with $E$ an effective $\mathbb{Q}$-divisor. In addition, the support of $E$ is a normal crossings divisor since $f$ is a log resolution. Choosing $d$ such that $n / d^{2}<\epsilon$ and pulling back to $X$, we have

$$
K_{X(d)}={\widetilde{\mu_{d}}}^{*} K_{X}={\widetilde{\mu_{d}}}^{*}\left(f^{*} K_{Y}-\frac{n}{d^{2}} M\right)+\frac{n}{d^{2}}{\widetilde{\mu_{d}}}^{*} M+{\widetilde{\mu_{d}}}^{*} E .
$$

The statement now follows by observing that

- $\left(n / d^{2}\right){\widetilde{\mu_{d}}}^{*} M=n M_{d}$;

- $L:={\widetilde{\mu_{d}}}^{*}\left(f^{*} K_{Y}-\left(n / d^{2}\right) M\right)={\widetilde{\mu_{d}}}^{*} f^{*} K_{Y}-n M_{d}$ is a nef and big line bundle;

- ${\widetilde{\mu_{d}}}^{*} E$ is an effective $\mathbb{Q}$-divisor with normal crossings support. 
Proof of Theorem 4.1. Since $X$ is of maximal Albanese dimension, $K_{X}$ is effective and $\left|2 K_{X}\right| \subseteq$ $\left|m K_{X}\right|$ for $m \geqslant 2$; hence, it is enough to prove the statement for $m=2$. We may also assume $n \geqslant 2$, since for $n=1$, the statement follows easily by the Riemann-Roch theorem.

By Theorem 2.2 and Lemma 4.3, we can fix a $d \gg 0$ such that

- $K_{X^{(d)}}=L+n M_{d}+E$, with $L$ nef and big and $E$ effective with normal crossings support;

- for $\alpha \in \operatorname{Pic}^{0}(A)=\operatorname{Pic}^{0}(X)$ general, the map given by $\left|2 K_{X^{(d)}}+\alpha\right|$ coincides with $\varphi^{(d)}$ (notation as in Theorem 2.2).

Write $E=\lfloor E\rfloor+\Delta$, so that $\Delta \geqslant 0$ is a $\mathbb{Q}$-divisor with $\lfloor\Delta\rfloor=0$ and normal crossings support. Set $D:=K_{X^{(d)}}-\lfloor E\rfloor=L+n M_{d}+\Delta$. We are going to show that if $C \subset X$ is the intersection of $(n-1)$ general elements of $\left|M_{d}\right|$, then the map induced by $\left|K_{X^{(d)}}+D+\alpha\right|$ restricts to a generically injective map on $C$ for $\alpha \in \operatorname{Pic}^{0}(A)$ general. Since $\left|K_{X^{(d)}}+D+\alpha\right| \subseteq\left|2 K_{X^{(d)}}+\alpha\right|$ and the map $\varphi^{(d)}$ is composed with the map $a_{d}: X^{(d)} \rightarrow A$ by Theorem 2.2, this will prove the statement.

First of all, we prove that $\left|K_{X^{(d)}}+D+\alpha\right|$ restricts to a complete system on $C$, by showing that $h^{1}\left(\mathcal{I}_{C}\left(K_{X^{(d)}}+D+\alpha\right)\right)=0$ for every $\alpha \in \operatorname{Pic}^{0}(A)$. To this end, we look at the resolution of $\mathcal{I}_{C}$ given by the Koszul complex:

$$
0 \rightarrow \mathcal{O}_{X^{(d)}}\left(-(n-1) M_{d}\right) \rightarrow \cdots \rightarrow \bigwedge^{2}\left(\mathcal{O}_{X^{(d)}}\left(-M_{d}\right)^{\oplus^{n-1}}\right) \rightarrow \mathcal{O}_{X^{(d)}}\left(-M_{d}\right)^{\oplus^{n-1}} \rightarrow \mathcal{I}_{C} \rightarrow 0
$$

Twisting (4.1) by $K_{X^{(d)}}+D+\alpha$, we obtain a resolution of $\mathcal{I}_{C}\left(K_{X^{(d)}}+D+\alpha\right)$ such that every term is a sum of line bundles numerically equivalent to a line bundle of the form

$$
K_{X^{(d)}}+D-i M_{d}=K_{X^{(d)}}+L+(n-i) M_{d}+\Delta
$$

for some $1 \leqslant i \leqslant n-1$. Since the hypotheses of Kawamata-Viehweg's vanishing theorem apply to each of these line bundles, it follows that $\mathcal{I}_{C}\left(K_{X^{(d)}}+D+\alpha\right)$ has a resolution of length $n-2$ such that all the sheaves appearing in the resolution have zero higher cohomology. Hence, the hypercohomology spectral sequence gives $h^{1}\left(\mathcal{I}_{C}\left(K_{X^{(d)}}+D+\alpha\right)\right)=0$, as claimed.

Finally, observe that by construction, $\left.\left(K_{X^{(d)}}+D+\alpha\right)\right|_{C}$ has degree at least $2 g(C)-2+M_{d}^{n}>$ $2 g(C)+1$; therefore, it is very ample.

\section{Examples, remarks and open questions}

In this section, we give some examples of the eventual behaviour of the paracanonical system; we keep the notation of Sections 2 and 3. All our examples are constructed as abelian covers; we use the notation and the general theory of [Par91].

Let $\Gamma$ be a finite abelian group, let $\Gamma^{*}:=\operatorname{Hom}\left(\Gamma, \mathbb{C}^{*}\right)$ be its group of characters, and let $\pi: X \rightarrow Y$ be a flat $\Gamma$-cover with $Y$ smooth and $X$ normal. One has a decomposition $\pi_{*} \mathcal{O}_{X}=$ $\oplus_{\chi \in \Gamma^{*}} L_{\chi}^{-1}$, where $L_{\chi}$ is a line bundle and $\Gamma$ acts on $L_{\chi}^{-1}$ via the character $\chi$. In particular, we have $L_{1}=\mathcal{O}_{Y}$.

Our main observation is the following.

Lemma 5.1. In the above setup, assume that $X$ and $Y$ are smooth of maximal Albanese dimension; denote the Albanese map by $\bar{a}: Y \rightarrow A$. If

(a) the map $\pi$ is totally ramified, that is, it does not factor through an étale cover $Y^{\prime} \rightarrow Y$ of degree $>1$;

(b) for every $1 \neq \chi \in \Gamma^{*}$, we have $h^{1}\left(L_{\chi}^{-1}\right)=0$; 


\section{M. Á. Barja, R. PArdini And L. Stoppino}

(c) there exists precisely one element $\bar{\chi} \in \Gamma^{*}$ such that $h_{a}^{0}\left(K_{Y}+L_{\bar{\chi}}\right)>0$;

then

(i) the map $a:=\bar{a} \circ \pi$ is the Albanese map of $X$;

(ii) the eventual paracanonical map $\varphi: X \rightarrow Z$ is composed with $\pi: X \rightarrow Y$;

(iii) if, in addition, $K_{Y} \otimes L_{\bar{\chi}}$ is eventually generically birational (for example, if a is generically injective), then $\pi$ is birationally equivalent to $\varphi$.

Proof. We freely use the notation of Section 2.

(i) First of all, by condition (b), we have

$$
q(X)=h^{1}\left(\mathcal{O}_{X}\right)=\sum_{\chi} h^{1}\left(L_{\chi}^{-1}\right)=q(Y) ;
$$

hence, the induced map $\operatorname{Alb}(X) \rightarrow A$ is an isogeny. On the other hand, the fact that $\pi$ is totally ramified implies that the map $\operatorname{Pic}^{0}(Y) \rightarrow \operatorname{Pic}^{0}(X)$ is injective. It follows that $\operatorname{Alb}(X) \rightarrow A$ is an isomorphism and the map $a=\bar{a} \circ \pi$ is the Albanese map of $X$.

(ii) Let $\pi^{(d)}: X^{(d)} \rightarrow Y^{(d)}$ be the $\Gamma$-cover induced by $\pi: X \rightarrow Y$, taking base change with $Y^{(d)} \rightarrow Y$. There is a cartesian diagram

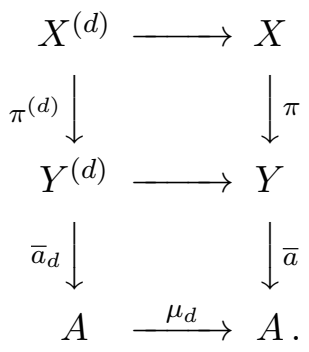

By Theorem 2.2, it is enough to show that for every $d$, the system $\left|K_{X^{(d)}} \otimes \alpha\right|$ is composed with $\pi^{(d)}: X^{(d)} \rightarrow Y^{(d)}$ for $\alpha \in \operatorname{Pic}^{0}(A)$ general or, equivalently, that $\left|K_{X^{(d)}} \otimes \alpha\right|$ is $\Gamma$-invariant for $\alpha \in \operatorname{Pic}^{0}(A)$ general. One has $\pi^{(d)}{ }_{*} \mathcal{O}_{X^{(d)}}=\oplus_{\chi \in \Gamma^{*}}\left(L_{\chi}^{(d)}\right)^{-1}$. Hence, by the formulae for abelian covers, we have

$$
h_{a}^{0}\left(K_{X^{(d)}}\right)=\sum_{\chi \in \Gamma^{*}} h_{a}^{0}\left(K_{Y^{(d)}} \otimes L_{\chi}^{(d)}\right)=h_{a}^{0}\left(K_{Y^{(d)}} \otimes L_{\bar{\chi}}^{(d)}\right)
$$

because of condition (c). So, for $\alpha \in \operatorname{Pic}^{0}(A)$ general, we have $H^{0}\left(K_{X^{(d)}} \otimes \alpha\right)=H^{0}\left(K_{Y^{(d)}} \otimes\right.$ $\left.L_{\bar{\chi}}^{(d)} \otimes \alpha\right)$; it follows that $\Gamma$ acts trivially on $\mathbb{P}\left(H^{0}\left(K_{X^{(d)}} \otimes \alpha\right)\right)$, and therefore the map given by $\left|K_{X^{(d)}} \otimes \alpha\right|$ factors through $\pi^{(d)}$.

(iii) The proof of statement (ii) shows that eventually $\varphi^{(d)}$ is birationally equivalent to the composition of $\pi^{(d)}$ with the map given by $\left|K_{Y^{(d)}} \otimes L_{\bar{\chi}}^{(d)} \otimes \alpha\right|$ for $\alpha \in \operatorname{Pic}^{0}(A)$ general. So if $\left|K_{Y} \otimes L_{\bar{\chi}}\right|$ is eventually generically birational, then $\varphi^{(d)}$ and $\pi^{(d)}$ are birationally equivalent for $d$ sufficiently large and divisible.

Example 5.2 (Examples with $m_{X}=2$ ). Every variety of maximal Albanese dimension $Z$ with $\chi(Z)=0$ and $\operatorname{dim} Z=n \geqslant 2$ occurs as the eventual paracanonical image for some $X$ of general type with $m_{X}=2$.

Let $L$ be a very ample line bundle on $Z$, pick a smooth divisor $B \in|2 L|$, and let $\pi: X \rightarrow Z$ be the double cover given by the pair $(L, B)$. Since $Z$ is of maximal Albanese dimension, $K_{Z}$ is 
effective, and therefore $\left|K_{Z} \otimes L\right|$ is birational. By Kodaira vanishing, we have $h^{1}\left(Z, L^{-1}\right)=0$ and

$$
\chi\left(K_{Z} \otimes L\right)=h^{0}\left(Z, K_{Z} \otimes L\right)=h_{a}^{0}\left(Z, K_{Z} \otimes L\right) .
$$

So, by continuity, the system $\left|K_{Z} \otimes L \otimes \alpha\right|$ is birational for general $\alpha \in \operatorname{Pic}^{0}(Z)$. So, the double cover $\pi: X \rightarrow Z$ satisfies conditions (a) and (b) of Lemma 5.1. Moreover, as, by assumption, $h_{a}^{0}\left(K_{Z}\right)=\chi(Z)=0$, condition (c) is also satisfied, and the eventual paracanonical map is composed with $\pi$. By [BPS16a, Lemma 3.4], the line bundle $K_{Z^{(d)}} \otimes L^{(d)}$ is generically birational for every $d$, and therefore $\pi: X \rightarrow Z$ is the eventual paracanonical map by Lemma 5.1(iii).

Remark 5.3. If in Example 5.2, we take a variety $Z$ whose Albanese map is not generically injective, then the eventual paracanonical map of the variety $X$ neither is an isomorphism nor coincides with the Albanese map of $X$.

Varieties $Z$ with $\chi(Z)=0$ whose Albanese map is generically finite of degree at least 2 do exist for $\operatorname{dim} Z \geqslant 2$. Observe that it is enough to construct 2-dimensional examples, since in higher dimension, one can consider the product of $Z$ with any variety of maximal Albanese dimension. To construct an example with $\operatorname{dim} Z=2$, one can proceed as follows:

- Take a curve $B$ with a $\mathbb{Z}_{2}$-action such that the quotient map $\pi: B \rightarrow B^{\prime}:=B / \mathbb{Z}_{2}$ is ramified and $g\left(B^{\prime}\right)>0$.

- Take an elliptic curve $E$, choose a $\mathbb{Z}_{2}$-action by translation, and denote the quotient curve $E / \mathbb{Z}_{2}$ by $E^{\prime}$.

- Set $Z:=(B \times E) / \mathbb{Z}_{2}$, where $\mathbb{Z}_{2}$ acts diagonally on the product.

The map $f: Z \rightarrow B^{\prime} \times E^{\prime}$ is a ramified double cover, and it is easy to see that the Albanese map of $Z$ is the composition of $f$ with the inclusion $E^{\prime} \times B^{\prime} \rightarrow E^{\prime} \times J\left(B^{\prime}\right)$. Indeed, by the universal property of the Albanese map, there is a factorization $Z \rightarrow \operatorname{Alb}(Z) \rightarrow E^{\prime} \times J\left(B^{\prime}\right)$. The map $\operatorname{Alb}(Z) \rightarrow E^{\prime} \times J\left(B^{\prime}\right)$ is an isogeny since $q(Z)=g\left(B^{\prime}\right)+1$; in addition, the map $E^{\prime} \times J\left(B^{\prime}\right) \rightarrow$ $\operatorname{Pic}^{0}(Z)$ between the dual abelian varieties is injective since $f$ is ramified. We conclude that $\operatorname{Alb}(Z) \rightarrow E^{\prime} \times J\left(B^{\prime}\right)$ is an isomorphism, and therefore the Albanese map of $Z$ has degree 2 .

Example 5.4 (Examples with $m_{X}=4$ and $\chi(Z)=0$ ). For $i=1,2$, let $A_{i}$ be abelian varieties of dimension $q_{i} \geqslant 1$, and set $A=A_{1} \times A_{2}$. For $i=1,2$, let $M_{i}$ be a very ample line bundle on $A_{i}$, and choose $B_{i} \in\left|2 M_{i}\right|$ general; we let $\pi: X \rightarrow A$ be the $\mathbb{Z}_{2}^{2}$-cover with branch divisors $D_{1}=\operatorname{pr}_{1}^{*} B_{1}$, $D_{2}=\operatorname{pr}_{2}^{*} B_{2}$ and $D_{3}=0$, associated with $\left(L_{1}, D_{1}\right)$ and $\left(L_{2}, D_{2}\right)$, where $L_{1}=\eta_{1} \otimes M_{2}$ and $L_{2}=M_{1} \otimes \eta_{2}$ with $0 \neq \eta_{i} \in \operatorname{Pic}^{0}\left(A_{i}\right)[2]$. One has $L_{3}=L_{1} \otimes L_{2}$, so that $L_{3}$ is very ample. It is easy to check that $h^{1}\left(L_{j}^{-1}\right)=0$ for $j=1,2,3$ and $h_{a}^{0}\left(L_{j}\right)>0$ if and only if $j=3$. So the assumptions of Lemma 5.1 are satisfied, and arguing as in Example 5.2, we see that $\pi: X \rightarrow A$ is both the Albanese map and the eventual paracanonical map of $X$.

An interesting feature of this example is that for $d$ even, the cover $\pi^{(d)}: X^{(d)} \rightarrow A^{(d)}=A$ is the product of two double covers $f_{i}^{(d)}: X_{i} \rightarrow A_{i}$, for $i=1,2$, branched on the pull-back of $B_{i}$. If $q_{1}, q_{2} \geqslant 2$, then $q\left(X^{(d)}\right)=q(X)$, but if, say, $q_{1}=1$, then $q\left(X^{(d)}\right)$ grows with $d$.

In particular, if $q_{1}=q_{2}=1$, then for $d$ even, the surface $X^{(d)}$ is a product of bielliptic curves.

Example 5.5 (Examples with large $m_{X}$ ). A variation of Example 5.4 gives examples with higher values of $m_{X}$. For simplicity, we describe an example of a threefold $X$ with $m_{X}=8$, but it is easy to see how to modify the construction to obtain an example with $m_{X}=2^{k}$ for every $k$ (the dimension of the examples will also increase, of course). 


\section{M. Á. Barja, R. PArdini And L. Stoppino}

For $i=1, \ldots, 3$, let $A_{i}$ be elliptic curves, let $M_{i}$ be a very ample divisor on $A_{i}$, and let $B_{i} \in\left|2 M_{i}\right|$ be a general divisor. Set $A=A_{1} \times A_{2} \times A_{3}$, and choose $\eta_{1}, \eta_{2} \in \operatorname{Pic}^{0}(A)[2]$ distinct such that, setting $\eta_{3}=\eta_{1}+\eta_{2}$, we have $\left.\eta_{j}\right|_{A_{i}} \neq 0$ for every choice of $i, j \in\{1,2,3\}$. Set $\Gamma:=\mathbb{Z}_{2}^{3}$, and denote the standard generators of $\Gamma$ by $\gamma_{1}, \gamma_{2}, \gamma_{3}$. We let $\pi: X \rightarrow A$ be the $\Gamma$-cover given by the following building data:

$$
D_{\gamma_{i}}=\operatorname{pr}_{i}^{*} B_{i}, \quad i=1, \ldots, 3, \quad D_{\gamma}=0 \quad \text { if } \quad \gamma \neq \gamma_{i},
$$

and

$$
L_{i}=\operatorname{pr}_{i}^{*} M_{i} \otimes \eta_{i}, \quad i=1, \ldots, 3 .
$$

It is immediate to see that the reduced fundamental relations (cf. [Par91, Proposition 2.1])

$$
2 L_{i} \equiv D_{\gamma_{i}}, \quad i=1, \ldots, 3
$$

are satisfied. The corresponding cover $\pi: X \rightarrow A$ satisfies the assumptions of Lemma 5.1, and arguing as in the previous cases, we see that $\pi: X \rightarrow A$ is both the Albanese map and the eventual paracanonical map of $X$.

Question 5.6. The statement of Theorem 3.1 shows an analogy between the eventual behaviour of the paracanonical system of varieties of maximal Albanese dimension and the canonical map (cf. [Bea79, Theorem 3.1]). As in the case of the canonical map, the previous examples show that it is fairly easy to produce examples of case (b) of Theorem 3.1 (cf. Example 5.2). On the other hand, we do not know any examples of case (a) with $m_{X}>1$.

For instance, in the 2-dimensional case, by Proposition 3.3, the variety $X$ would be a surface of general type and maximal Albanese dimension with an involution $\sigma$ such that $q(X / \sigma)=q(X)$ and $p_{g}(X / \sigma)=p_{g}(X)$ and such that the eventual paracanonical map of $X / \sigma$ is birational. If such an $X$ exists, then its Albanese map is of degree $2 k$, with $k \geqslant 2$. Indeed, the Albanese map of $X$ is composed with $\sigma$ and $K_{X / \sigma}^{2} \leqslant \frac{1}{2} K_{X}^{2} \leqslant \frac{9}{2} \chi(X)=\frac{9}{2} \chi(X / \sigma)<5 \chi(X / \sigma)$, where the last but one inequality is given by the Bogomolov-Miyaoka-Yau inequality. Proposition 6.14 of [BPS16a] implies that the Albanese map of $X / \sigma$ has degree $k>1 .^{1}$

Question 5.7. In the surface case, the eventual paracanonical degree is at most 4 by Proposition 3.3. In order to give a bound for higher-dimensional varieties in the same way, one would need to bound the volume of $K_{X}$ in terms of $\chi\left(K_{X}\right)$; however, [BPS16a, Example 8.5] shows that this is not possible for $n \geqslant 3$. So, it is an open question whether it is possible to give a bound on $m_{X}$, for $X$ a variety of fixed dimension $n>2$. Note that Example 5.5 shows that this bound, if it exists, has to increase with $n .^{2}$

\section{ACKNOWLEDGEMENTS}

We wish to thank Christopher Hacon for very useful mathematical communications, and especially for pointing out the reference [HM07]. The second- and third-named authors would like to thank the Departament de Matemàtiques of the Universitat Politècnica de Catalunya for the invitation and the warm hospitality.

\footnotetext{
${ }^{1}$ While this paper was under revision, the preprint [Jia16] appeared, where it is proven that such a surface does not exist.

${ }^{2}$ This question has been partly answered in the preprint [Jia16] for the case of threefolds.
} 


\section{The EVENTUAL PARACANONICAL MAP}

\section{REFERENCES}

Bar15 M. A. Barja, Generalized Clifford-Severi inequality and the volume of irregular varieties, Duke Math. J. 164 (2015), no. 3, 541-568; doi:10.1215/00127094-2871306.

BCHM10 C. Birkar, P. Cascini, C.D. Hacon and J. McKernan, Existence of minimal models for varieties of log general type, J. Amer. Math. Soc. 23 (2010), no. 2, 405-468; doi:10.1090/S0894-0347-09-00649-3.

Bea79 A. Beauville, L'application canonique pour les surfaces de type général, Invent. Math. 55 (1979), no. 2, 121-140; doi:10.1007/BF01390086.

BL04 C. Birkenhake and H. Lange, Complex abelian varieties, 2nd ed., Grundlehren math. Wiss., vol. 302 (Springer-Verlag, Berlin, 2004); doi:10.1007/978-3-662-06307-1.

BLNP12 M. A. Barja, M. Lahoz, J. C. Naranjo and G. Pareschi, On the bicanonical map of irregular varieties, J. Algebraic Geom. 21 (2012), no. 3, 445-471; doi:10.1090/S1056-3911-2011-00565-1.

BPS16a M. A. Barja, R. Pardini and L. Stoppino, Linear systems on irregular varieties, J. Inst. Math. Jussieu (to appear), arXiv:1606.03290.

BPS16b_ Surfaces on the Severi line, J. Math. Pures Appl. 105 (2016), no. 5, 734-743; doi:10.1016/j.matpur.2015.11.012.

CCZ07 J.A. Chen, M. Chen and D.-Q. Zhang, The 5-canonical system on 3-folds of general type, J. reine angew. Math. 603 (2007), 165-181; doi:10.1515/CRELLE.2007.015.

CH02 J.A. Chen and C.D. Hacon, Linear series of irregular varieties, Algebraic Geometry in East Asia (Kyoto, 2001) (World Sci. Publ., River Edge, NJ, 2002), 143-153; doi:10. 1142/9789812705105_0004.

EL97 L. Ein and R. Lazarsfeld, Singularities of theta divisors and the birational geometry of irregular varieties, J. Amer. Math. Soc. 10 (1997), no. 1, 243-258; doi:10.1090/ S0894-0347-97-00223-3.

HM07 C. D. Hacon and J. Mckernan, On Shokurov's rational connectedness conjecture, Duke Math. J. 138 (2007), no. 1, 119-136; doi:10.1215/S0012-7094-07-13813-4.

Jia16 Z. Jiang, Some results on the eventual paracanonical maps, 2016, arXiv:1611.07141.

JLT13 Z. Jiang, M. Lahoz and S. Tirabassi, On the Iitaka fibration of varieties of maximal Albanese dimension, Int. Math. Res. Not. 2013 (2013), no. 13, 2984-3005; doi:10.1093/imrn/rns131.

Par91 R. Pardini, Abelian covers of algebraic varieties, J. reine angew. Math. 417 (1991), 191-213; doi:10.1515/crll.1991.417.191.

PP11 G. Pareschi and M. Popa, Regularity on abelian varieties III: relationship with generic vanishing and applications, Grassmannians, Moduli Spaces and Vector Bundles, Clay Math. Proc., vol. 14 (Amer. Math. Soc., Providence, RI, 2011), 141-167.

Miguel Ángel Barja miguel.angel.barja@upc.edu

Departament de Matemàtiques, Universitat Politècnica de Catalunya, Avda. Diagonal 647, 08028

Barcelona, Spain

Rita Pardini rita.pardini@unipi.it

Dipartimento di Matematica, Università di Pisa, Largo B. Pontecorvo 5, I-56127 Pisa, Italy

Lidia Stoppino lidia.stoppino@uninsubria.it

Dipartimento di Scienza e Alta Tecnologia, Università dell'Insubria, Via Valleggio 11, 22100, Como, Italy 